\title{
A FEASIBILITY STUDY ABOUT THE EFFECTIVENESS OF IN SITU ELECTROKINETIC REMEDIATION (EKR) USING ELECTRICAL RESISTIVITY METHOD (ERM)
}

\author{
ANIS AYUNI SUIED*1, SAIFUL AZHAR AHMAD TAJUDIN² AND AZIMAN MADUN² \\ ${ }^{1}$ Research for Soft Soil Centre (RECESS), Universiti Tun Hussein Onn Malaysia. ${ }^{2,3}$ Faculty of Civil Engineering and \\ Environmental, Universiti Tun Hussein Onn Malaysia, 86400 Parit Raja, Batu Pahat, Johor Darul Takzim. \\ *Corresponding author: ayunisuied@gmail.com \\ Submitted final draft: 30 November 2020 Accepted: 9 January 2021 \\ http://doi.org/10.46754/jssm.2021.10.003
}

\begin{abstract}
Heavy metal contamination in soil has become a global issue that demands adequate attention and resolution. The related soil contaminant may affect human health and other living organisms in the ecosystem. Electrokinetic Remediation (EKR), which is a green technology-based approach that promotes a sustainable ecosystem, can be be used to resolve soil contaminated with heavy metals. Ex situ analysis has shown that more than 50 percent of electrokinetic remediation successfully removes heavy metals in soil. Hence, it is crucial that in situ remediations be implemented in field and environmental contexts. However, there are some challenges and a lack of guidelines on the field application of in situ remediations. This paper aims to propose a design guideline on in situ remediation application in field and environmental contexts based on a pilot study. The electrokinetic process of uncontaminated soil was conducted using a pure system. Preliminary data based on electrical resistivity tomography (ERT) before and after the remediation process were also applied.
\end{abstract}

Keywords: Heavy metal, Electrokinetic Remediation (EKR), Electrical Resistivity Tomography (ERT).

\section{Introduction}

Land may be contaminated when it contains a sufficient amount of toxic or harmful material that poses a threat to the health and safety of users of the land or workers engaged in its redevelopment. Similarly, the integrity of buildings and plants may also be at risk. Due to rapid industrialization and urbanization, there has been a considerable increase in the discharge of industrial waste into the ecosystem, leading to the accumulation of heavy metals in the soil, groundwater, and surface water (Sruthy et. al., 2014). Basically, some metals are required by plants in tiny amounts for their growth and optimum performance. However, many of these metals even in low concentration are highly toxic to the environment. Some of these metals, such as arsenic, cadmium, chromium, copper, lead, mercury, nickel, selenium, silver and zinc, are especially harmful because of their high level of toxicity. Furthermore, these metals are especially dangerous because they cannot be broken down quickly, as well as them having a long-lasting effect on the ecosystem and being responsible for significant health concerns worldwide (Dixit et al., 2015).

Due to population growth, the increasing number of landfills represents a growing threat to the environment and public health (air and groundwater pollution) (Dumont et. al., 2016). Similarly, the production of fly ash from power plant operations contains heavy metal toxicity, which causes groundwater and surface water pollution as well as presents a high risk to aquatic habitats and wildlife (Lemly, 2017).

\section{Electrokinetic Remediation (EKR)}

According to previous research (Mosavat, 2012), in comparison to conventional remediation technologies, EKR is cost-effective, and applicable to in situ and ex situ, is easy to install and operate (simplicity), has a silent operation, has the benefit of not disrupting site activities and has a relatively short treatment duration. To achieve high efficiency in the 
application of the EKR technique, the soil samples need to have a high concentration of heavy metal ions in their metallic state to make sure the process is successfully dissolved and that the heavy metal can be separated from the soil sample. The electrokinetic remediation is mainly characterized by cost-effectiveness in terms of its soil properties, such as the depth of contamination, cost of placing treatment zones, clean-up time and cost of labour and electrical power (Virtutyte et al., 2002). The higher voltage that is applied to the soil may increase the temperature of the soil which aids the electrokinetic process (Balia, 2018). However, the efficiency of the treatment may decrease due to temperature (Virtutyte et al., 2002). Moreover, the parameters of EKR also depends on the current supply, voltage drop, $\mathrm{pH}$ value, electrolyte concentration, ionic concentration, and time duration for the migration of heavy metal towards the anode and cathode based on its ionic charge (Athmer \& Ho, 2009; Azhar et al., 2016a; 2016b; Cameselle \& Pena 2016; Elicker et al., 2014; Hamdan \& Reddy, 2008). Presently, the current state of research is limited to the remediated artificially contaminated soil in the laboratory. However, the future research landscape is expected to focus on the field application of EKR technique (Chaudhary et al., 2017). Therefore, it is essential that more attention is given to the evaluation method for in-situ remediation assessment (Song et al., 2017).

\section{Methodology}

The methodology provides a rigorous approach and guideline needed to achieve the objective of a research based on a specified strategy. Figure 1 summarizes the procedures into a flowchart of in-situ electrokinetic remediation (EKR). Firstly, the researcher identified the efficient field application based on previous studies and fundamental theory. Because the researcher aims to compare the electrical resistivity tomography (ERT) in a pilot study to provide design guidelines for in-situ remediations, therefore, ERT was categorized into 3 main types of mapping which includes:

A. Preliminary mapping as a reference for soil mapping,

B. Remediation mapping aims to compare the duration for electro-osmosis process.

C. Post-remediation aims to justify the obtained results.

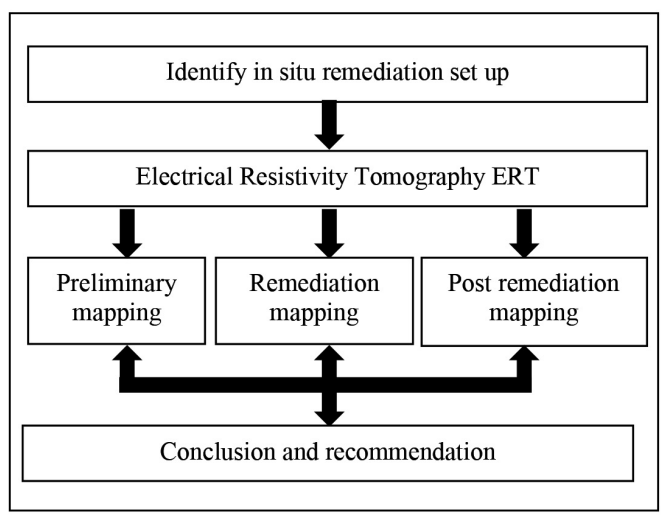

Figure 1: Flowchart of in situ electrokinetic remediations (EKR)

\section{Identify In Situ Remediation Set Up}

The electrokinetic process application will present the in-situ remediation under real environmental conditions. Basically, in-situ remediations was redesigned and enhanced from (Suied et al., 2018) where the remediation spacing was scaled up to 400 centimetres between the anode and cathode terminal as illustrated in Figure 2. The electrode terminal was made of stainless steel which is connected to the power supply.

The electrolyte tank was installed in an artificial embankment as shown in Figure 3. The artificial embankment was built using real clay soil from Ayer Hitam, Johor (an area classified as a restricted research area). Therefore, there are no requirements for original soil or groundwater. Other equipment required to initiate the electric supply throughout the electrokinetic process includes the power supply and crocodile clipper ( 2 meters in length) as shown in Figure 4. 


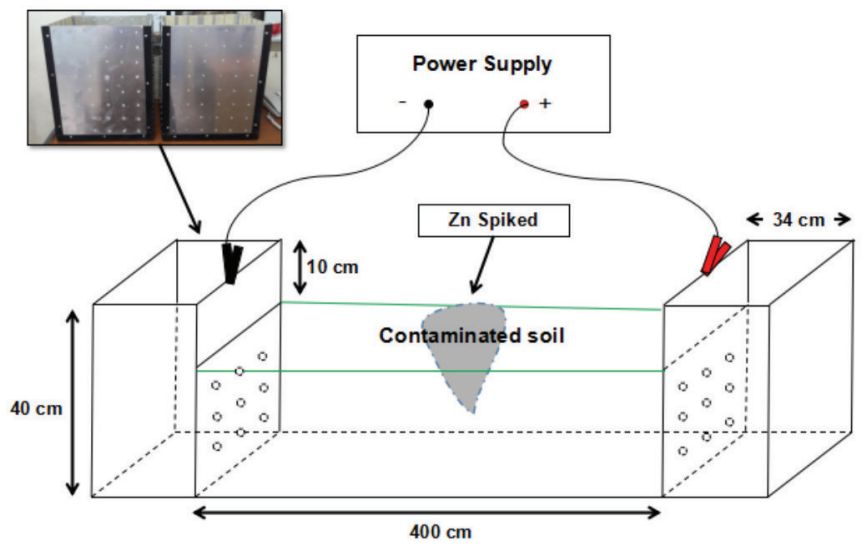

Figure 2: Schematic diagram for Electrokinetic Remediation (EKR) set up

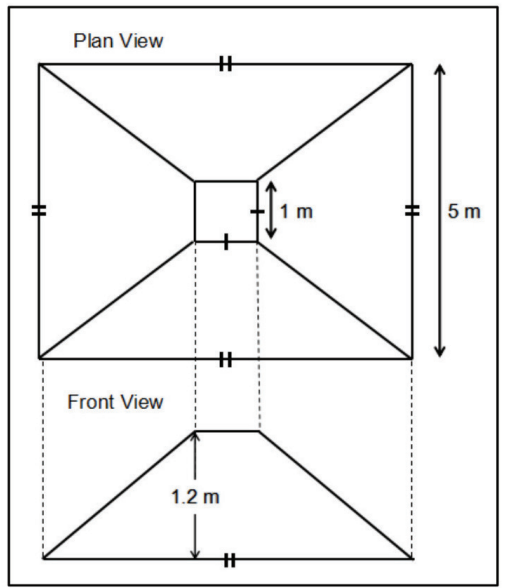

Figure 3: Schematic Diagram of EKR set up on Artificial Embankment
The electrolyte is one of the vital elements that is used to generate the electroosmotic process. However, the electrode tank was mentioned in Suied et al. (2018) as shown in Figure 4(a). The approach shown in Figure 4(a) was impractical and inconvenient. Hence, the preliminary investigation successfully demonstrated that the electrode tank causes electrolyte leakage and this leakage was absorbed into the soil as shown in Figure 4 (b).

Since the electrolyte tank is unable to hold the electrolyte, this study identified the solution for electrolyte compartment using a soil box of ex situ remediation (Jo et al., 2012; Rojo et al., 2012; Suzuki et al., 2013) which can be scaled up for field application for in situ

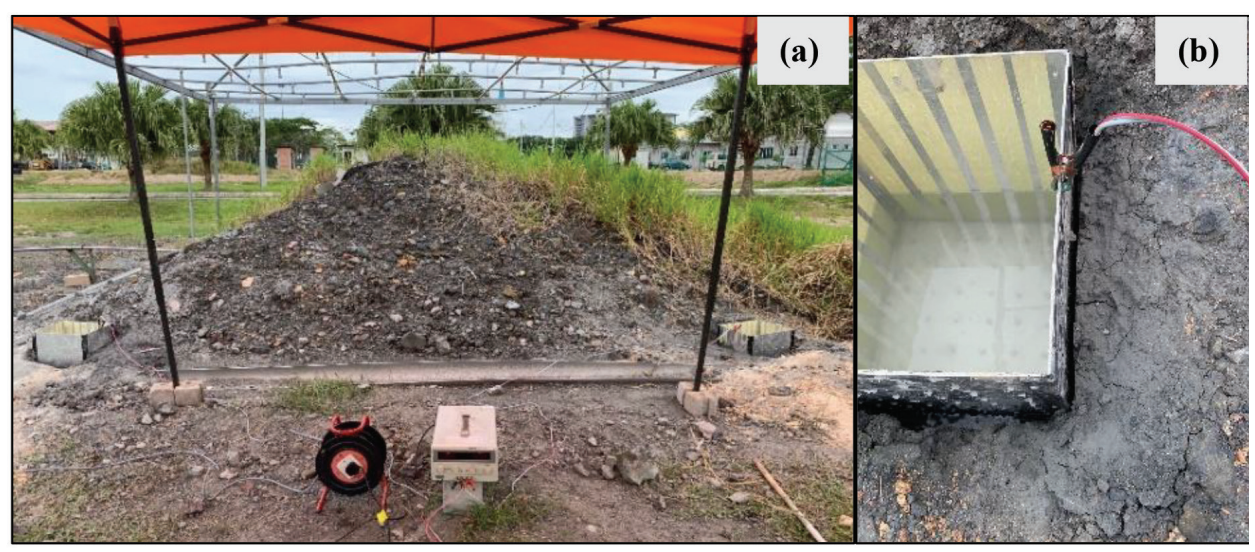

Figure 4: (a) In Situ Electrokinetic Remediation (EKR) set up using electrolyte tank; (b) Cracking of Electrolyte tank installed into the soil 
remediations. Therefore, cementation is one of the approaches that can be adopted for the electrolyte compartment in field application. The electrolyte compartment was covered with a cement surface except for the surface opposite the anode and cathode terminal. The soil surface was attached with a stainless-steel plate to promote the electric field towards the electroosmosis process as demonstrated in Figure 5. In addition, the stainless steel was perforated and covered with filter paper. This was done to enhance the electro-osmotic flow. Figure 6 shows the in situ remediation application successfully connected to a 100 Volt power supply.

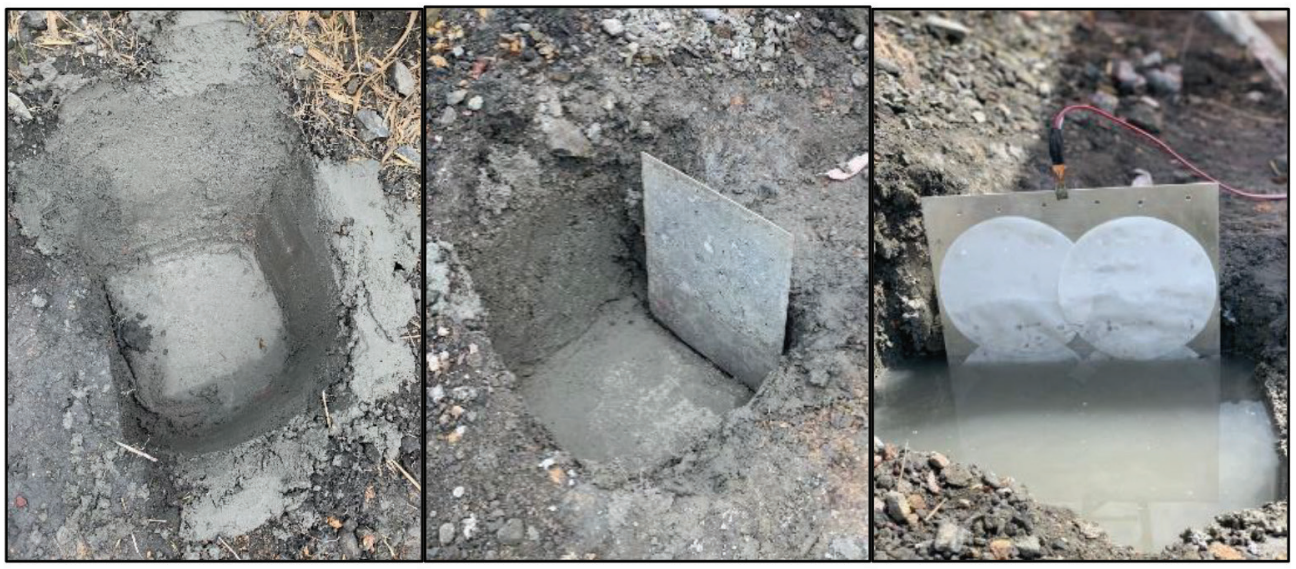

Figure 5: Electrolyte compartment that attached to stainless steel

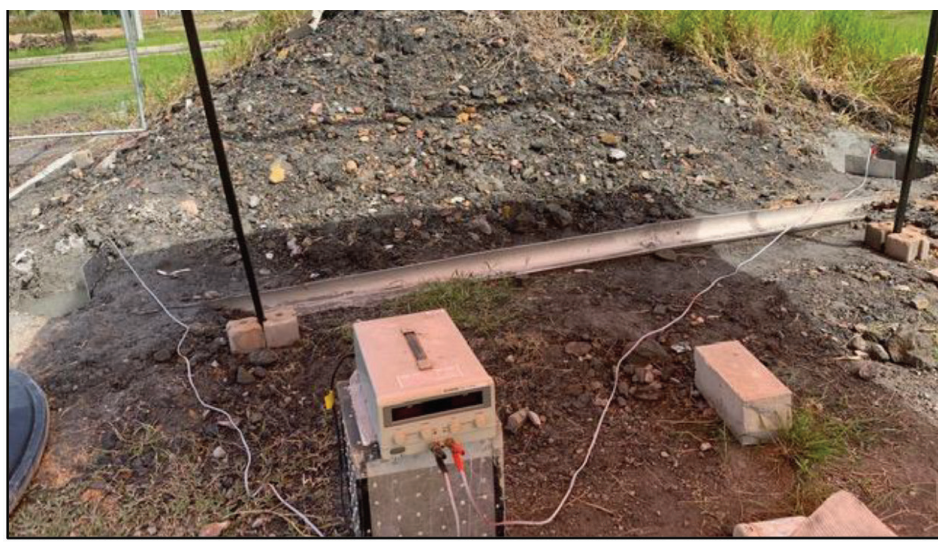

Figure 6: In situ electrokinetic remediation (EKR) set up using cementation method as electrolyte compartment

\section{Electrical Resistivity Tomography (ERT)}

The electrical resistivity method is one of the approaches used in environmental studies. Hence, the resistivity set up was applied as shown in Figure 7 below. The soil mapping was measured at the inclined surface of the embankment. Therefore, in Table 1 the recorded details of related equipment, such as the ABEM Terrameter LS2 (as shown in Figure 8), the modified electrode (16 $\mathrm{cm}$ in length), land cable and other accessories are shown. The measurement was analysed using RES2DINV software for electrical resistivity tomography (ERT). 


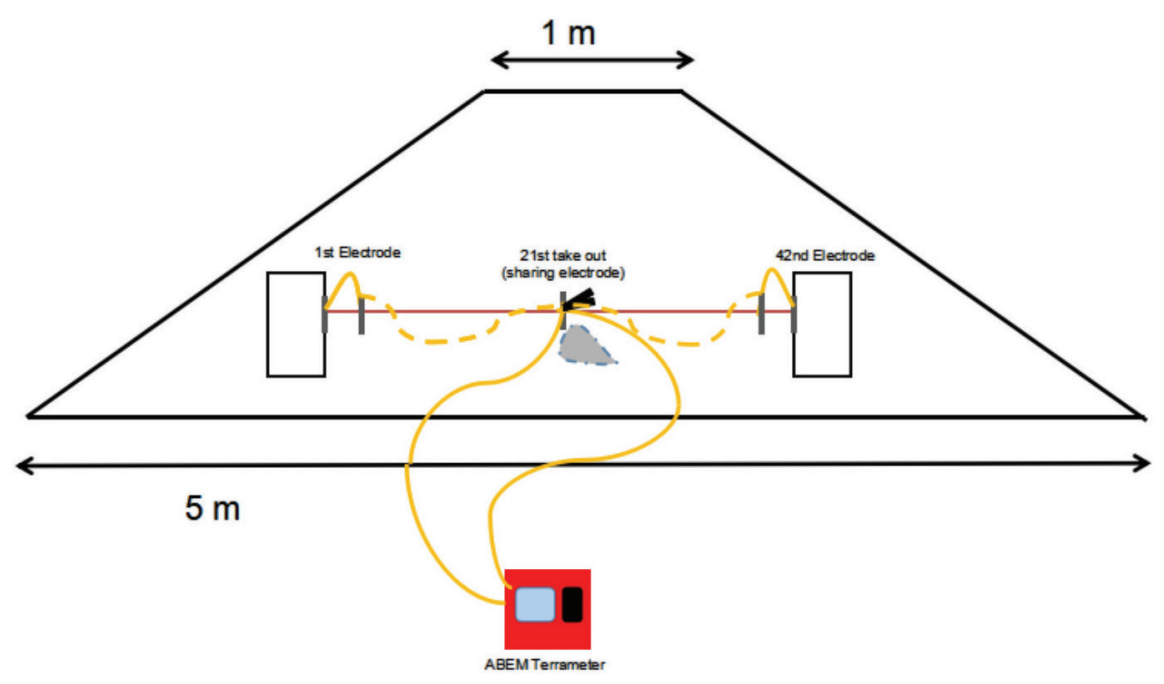

Figure 7: Schematic diagram for resistivity set up

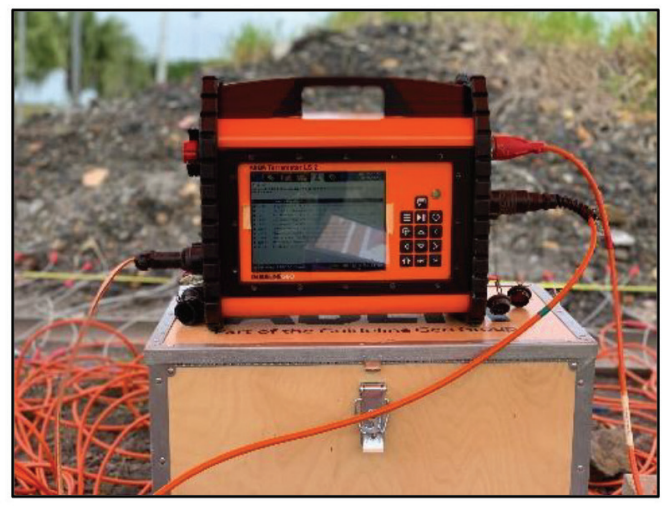

Figure 8: ABEM Terrameter LS2

\section{Results and Discussion}

\section{Preliminary Mapping}

The electrical resistivity method (ERM) was conducted at the artificial embankment as shown in Figure 7, which was built from original soil from Ayer Hitam, Johor, as shown in Figure 9. The electrical resistivity imaging (ERI) in a previous study by (Azhar et al., 2016) underlined that the slope could be categorized into two main zones and the resistivity values range between (i) Dry shale zone (600-2000 $\Omega . m$ ) and (ii) Shale zone near groundwater (20-600 $\Omega . \mathrm{m})$. For the electrical resistivity tomography (ERT)

Table 1: Electrical resistivity equipment

\begin{tabular}{lc}
\hline \multicolumn{1}{c}{ Equipment } & Value/Quantity \\
\hline ABEM Terrameter LS2 & 1 unit \\
Stainless steel Electrode & 42 unit \\
Electrode spacing & $10 \mathrm{~cm}$ \\
Land Cable & 2 unit \\
Jumper cable & 42 unit \\
Battery 12V & 2 unit \\
Rubber Hammer & 2 unit \\
Software & Terrameter Toolbox, RES2DINV \\
Data Transaction & 1 unit of Dongle \\
Adapter cable (for laptop) & 1 unit \\
\hline
\end{tabular}


Table 2: The time duration of the array set up

\begin{tabular}{ccc}
\hline Protocol Array & Number of Datum Point & Time Estimation \\
\hline Wenner & 190 & 45 minutes \\
Schlumberger & 524 & 2 hours 30 minutes \\
\hline
\end{tabular}

recorded in Figure 10, the Schlumberger array has a high potential for vertical resolution for a depth of 40 meters. Moreover, the ERT shown in Figure 11 is the Wenner array for horizontal resolution with a shallow depth of 35 meters.

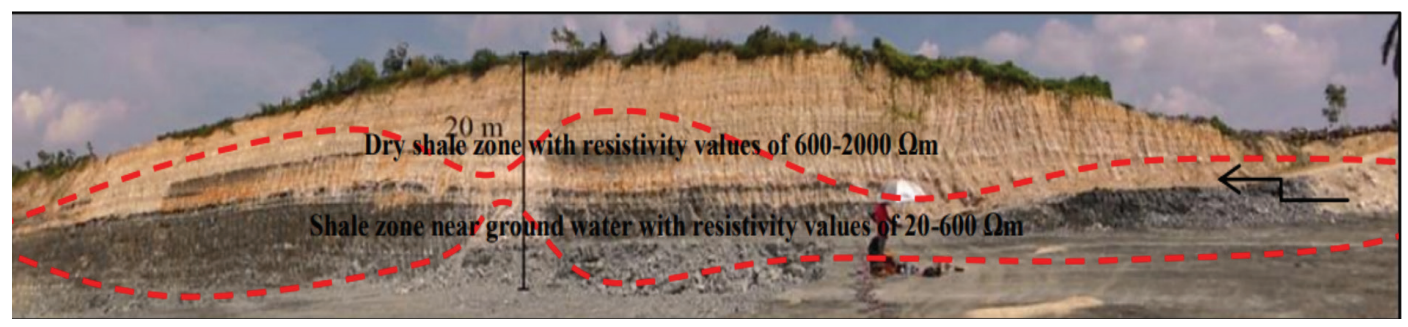

Figure 9: Soil slope located in Ayer Hitam Johor (Azhar et al., 2016)

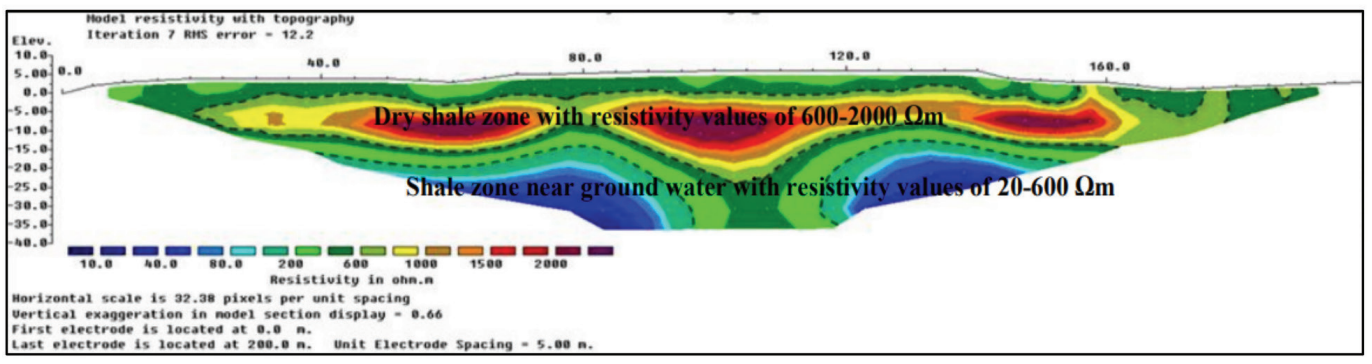

Figure 10: Electrical Resistivity Tomography (ERT) based on Schlumberger array

(Azhar et al., 2016)

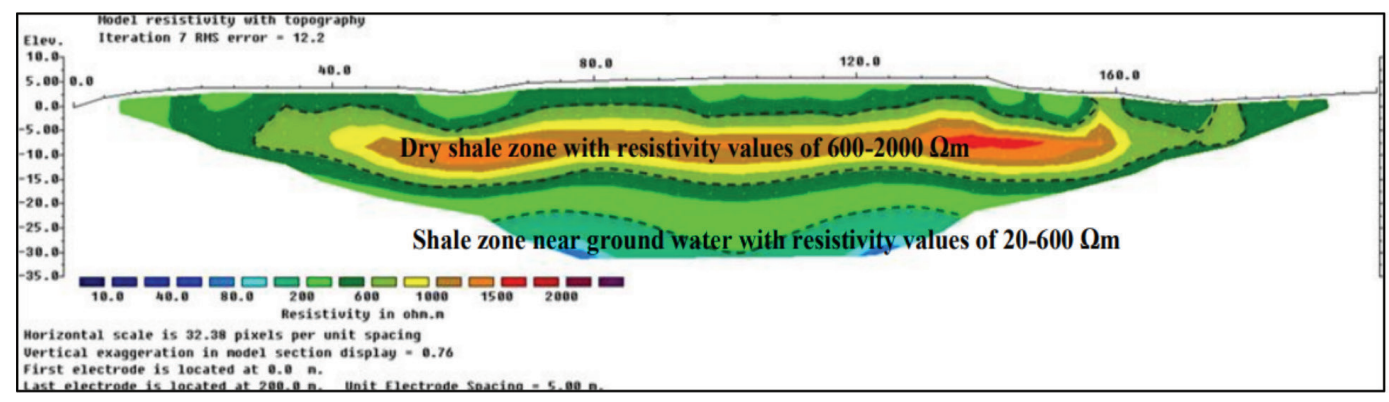

Figure 11: Electrical Resistivity Tomography (ERT) based on Wenner array

(Azhar et al., 2016)

The artificial embankment was extracted from the shale zone where preliminary analysis was extended to compare between both arrays, as shown in Figure 12 and Figure 13. The soil tomography illustrated that the artificial embankment is relatively in the range of 20 -

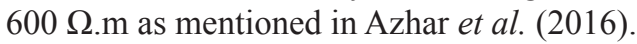


The resistivity value was slightly reduced due to equipment differences, electrode spacing, environmental factors and more. The equipment used in previous research for soil slope in Ayer
Hitam, Johor, were ABEM Terrameter SAS 4000 while the artificial embankment analysis used the ABEM Terrameter LS2.

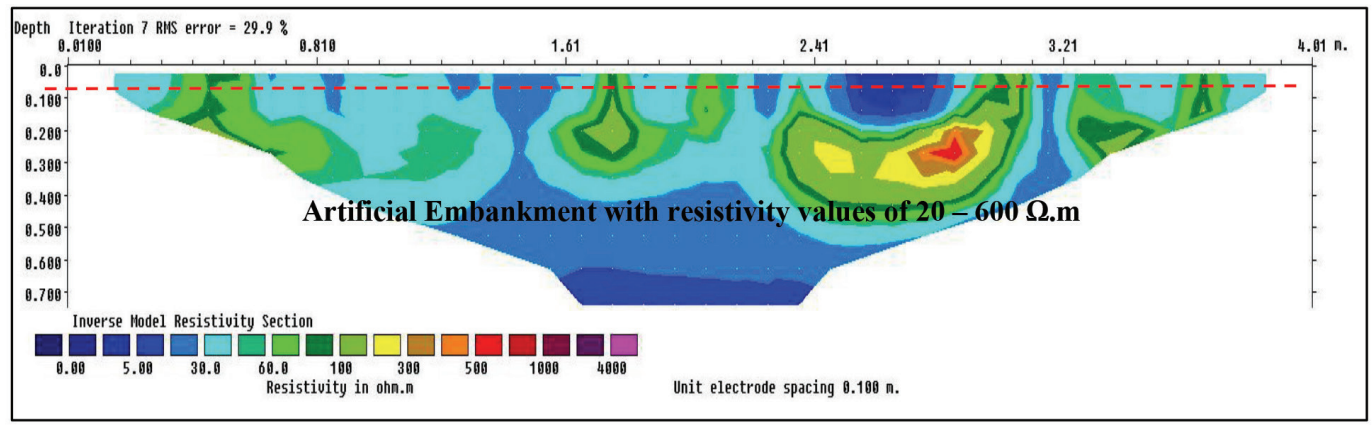

Figure 12: Electrical Resistivity Tomography (ERT) for artificial embankment based on Wenner array

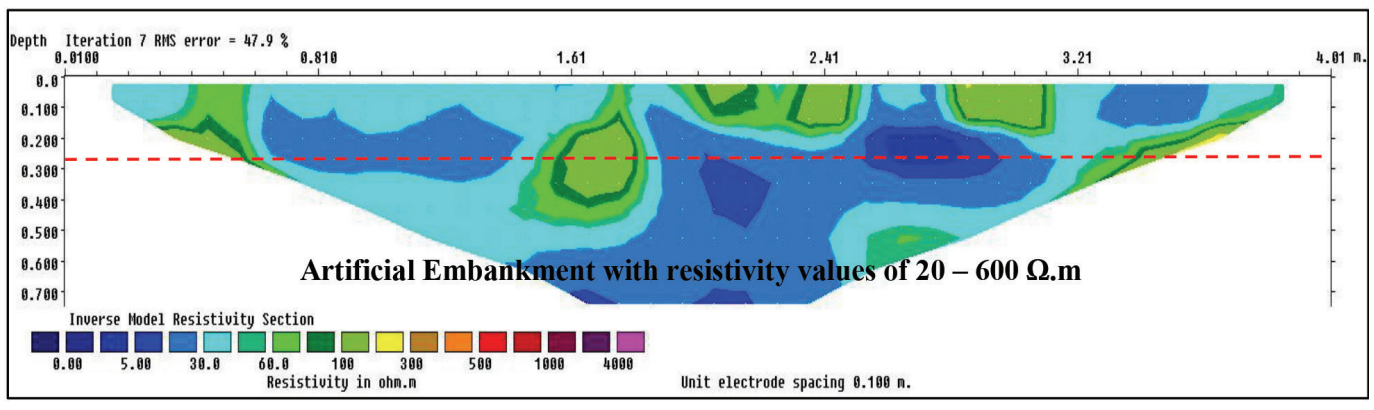

Figure 13: Electrical Resistivity Tomography (ERT) for artificial embankment based on Schlumberger array

Based on the specification for electrokinetic remediation (EKR), the electricity supply flows across the soil medium horizontally. Moreover, the stainless-steel plate was inserted into $30 \mathrm{~cm}$ of the electrolyte. Based on detailed Figures, the in-situ remediation was expected to provide an analysis for $30 \mathrm{~cm}$ to $40 \mathrm{~cm}$ depth. By comparing Figure 12 and Figure 13 above, the datum point had been covered up to 0.70 meters for both arrays. Therefore, the Wenner array should be precisely analysed since time constraint was one of the factors that determined the successful integration of the EKR and ERM methods as mentioned in Table 2.

Moisture Content is one of the parameters for electrical resistivity value (Azhar et al., 2017). Therefore, a preliminary analysis was conducted to determine the potential of the soil in absorbing water, which is poured on the soil surface. The resistivity value for the artificial embankment was measured at about half an hour. Based on the preliminary analysis, results show that this study can be limited to the Wenner array as a relatively horizontal resolution. As demonstrated in the previous mapping in Figure 12 , there is soil tomography based on 190 datum points as references. In order to determine the variation of water content that is absorbed into the soil, Figure 14 shows that the resistivity value dropped enormously due to the absorption of water. For the depth of $0.1 \mathrm{~m}$ until $0.2 \mathrm{~m}$ for both tomography, there is an apparent reduction in the resistivity value from $0.1-100 \Omega$.m to resistivity value of $0.1-60 \Omega$.m. In short, there was a 40 percent decrease in resistivity value after an hour of water absorption into the ground. 


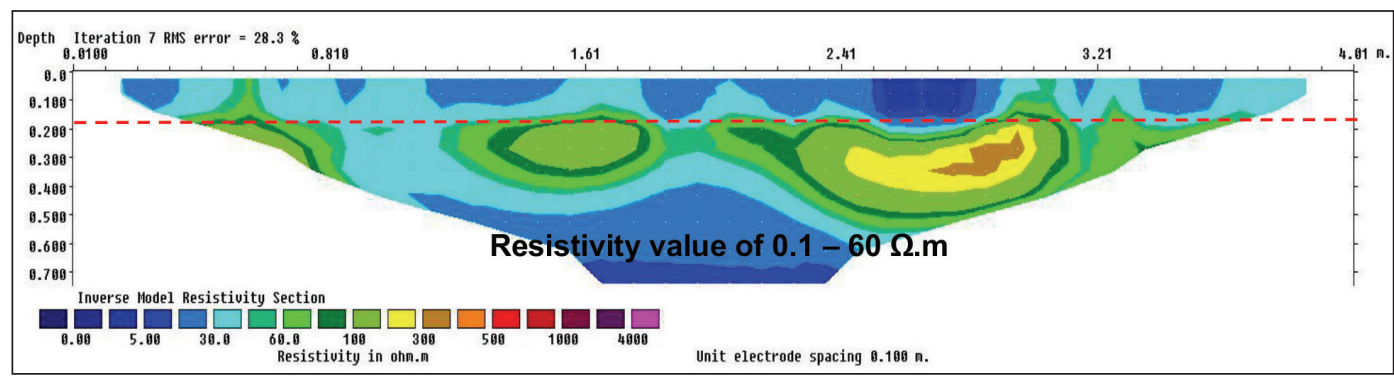

Figure 14: Electrical Resistivity Tomography (ERT) based on Wenner array for moisture soil

\section{Remediation Mapping}

In situ remediation was also conducted using a pure system with distilled water (DW) as an electrolyte. Figure 12 demonstrated the soil tomography for uncontaminated clayey soil in the artificial embankment. In Figure 12, there are three main areas that had been categorized as the anode, the middle and the cathode. Firstly, the researcher aimed to observe the electroosmotic process after two hours of movement of water from the anode to the cathode terminal as shown in Figure 15. At the anode and cathode terminals, the electrical resistivity value (ERV) decreased for more than 40 percent. However, the ERV remained unchanged in the middle area. Hence, it can be concluded that the remediation requires more than two hours for the water molecules to move under the electric field. Therefore, the remediation proceeded for 8 hours.

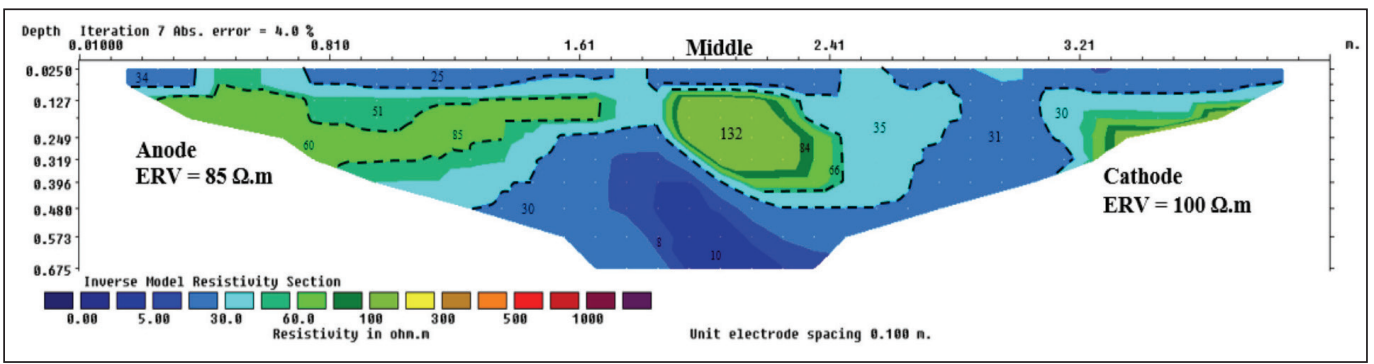

Figure 15: Electrical Resistivity Tomography (ERT) based on Wenner array before remediation

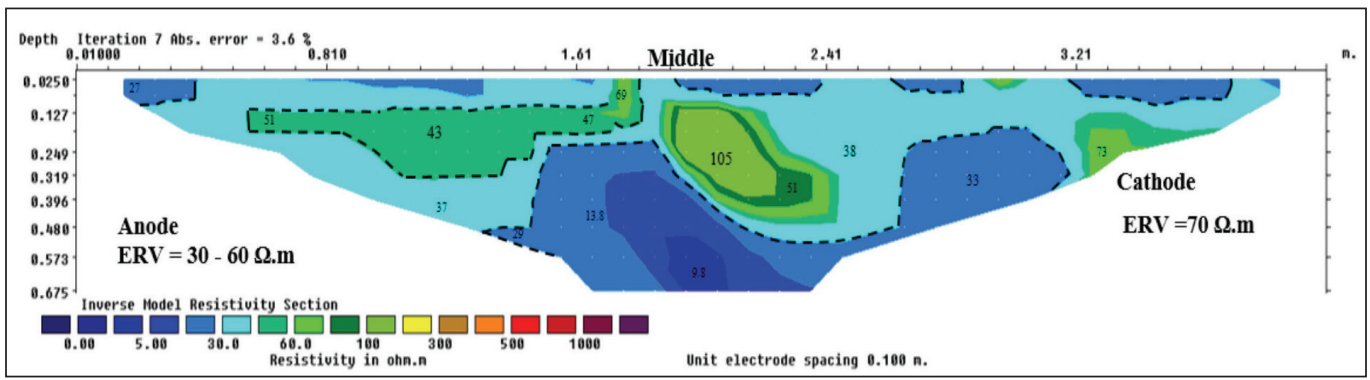

Figure 16: Electrical Resistivity Tomography (ERT) based on Wenner array after 2 hours of remediation 
Figure 16 shows the soil tomography for 8 hours of remediation. Based on observation during the remediation process, it was recorded that the electrolyte level remained unchanged from 5 to 8 hours. The ERV, on the other hand, increased drastically to a range of $100 \Omega$.m due to the electricity supply to the anode, middle and cathode areas. This occurred as a result of the electrokinetic process which supplied 100 to 110 Volts. Hence, it proves that it is adequate to remediate 400 meters of contaminated area. However, water occupied the air void inside the soil medium, resulting in no more water movement from the electrolyte. Moreover, the water content in the soil surface may dry in environmental conditions.

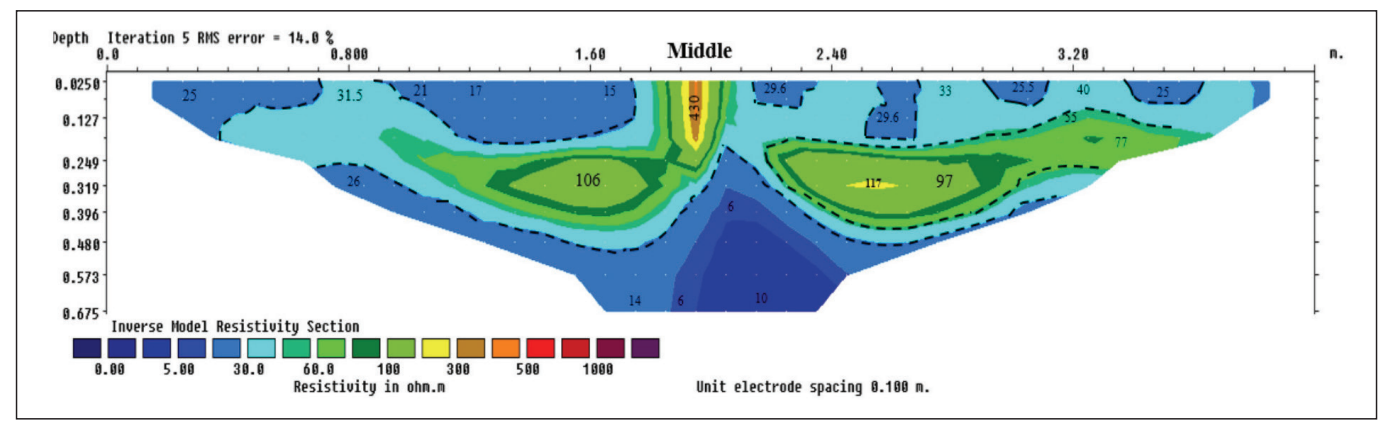

Figure 17: Electrical Resistivity Tomography (ERT) based on Wenner array after 8 hours of remediation

\section{Post-remediation Mapping}

Figure 18 also shows that the electric field can be discharged from the clayey soil when the tomography is altered back to a previous mapping after 24 hours remediation had been completed. Therefore, the electrokinetic remediation (EKR) with high potential can be successfully done under high moisture of soil types such as clay. However, field application will lead to environmental factors, such as hot weather absorbing the moisture. Therefore, during remediation, the soil needs extra support to ensure that the soil continuously has high moisture.

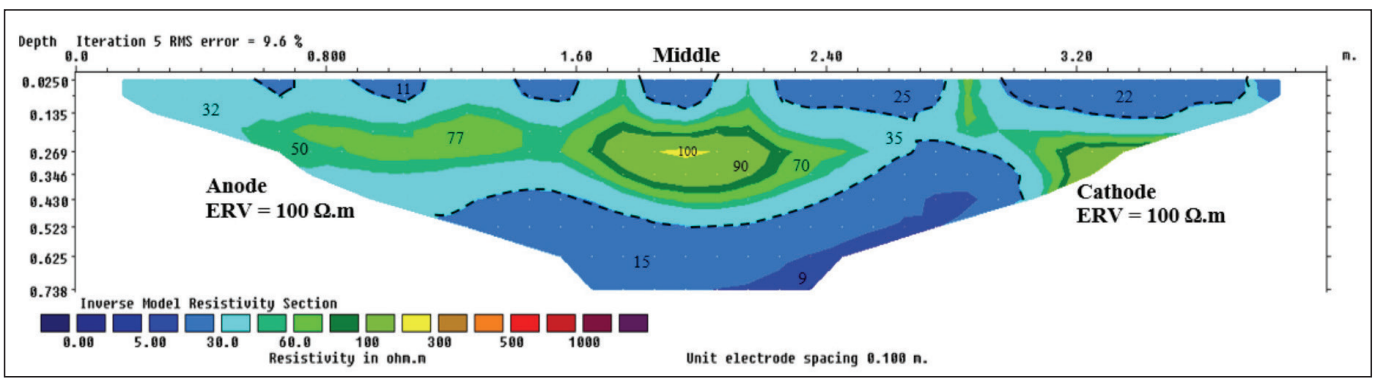

Figure 18: Electrical Resistivity Tomography (ERT) based on Wenner array after 24 hours discharge from remediation

\section{Conclusion}

In this study, electrokinetic remediation set up was developed to remediate clay soil for $400 \mathrm{~cm}$ in length and $30 \mathrm{~cm}$ in depth. The electrolyte should be adequately supplied to the contaminated area. In addition, the moisture of the soil surface should be maintained for more than 3 hours of remediation. Other than distilled water (DW) being used as an electrolyte in pure systems, the electrokinetic process and remediation can be enhanced using chelating agents, such as nitric acid, EDTA and more. 


\section{Acknowledgements}

This paper is financially funded by the MyBrain15 scholarship scheme under the Ministry of Higher Education Malaysia. The author would like to thank Preston Geochem Sdn. Bhd. for the collaboration.

\section{References}

Athmer, C. J., \& Ho, S. V. (2009). Field studies: Organic-contaminated soil remediation with Lasagna technology. In Reddy, K. R., \& Camesettle, C. (Eds.), Electrochemical remediation technologies for polluted soils, sediments, groundwater (pp 625-646). New Jersey: Wiley.

Azhar, A. T. S., Ayuni, S. A., Ezree, A. M., Nizam, Z. M., Aziman, M., Hazreek, Z. A. M., Norsuhaila, M. S., \& Zaidi, E. (2017). The use of Electrical Resistivity Method to mapping the migration of heavy metal by electrokinetic. IOP Conferences Series: Material Sciences and Engineering 226 (012061). IOP Publishing.

Azhar A. T. S., Hazreek Z. A. M., Aziman, M. Haimi D. S., \& Hafiz Z. M. (2016). Acidic Barren Slope Profiling using Electrical Resistivity Imaging (ERI) at Ayer Hitam, Johor. Journal of Physics: Conference Series 710. IOP Publishing.

Azhar, A. T. S., Nordin, N. S., Marto, A., Madun, A., Abidin, H. Z. A., Ian, J., \& Azmi, M. A. M. (2016a). The monitoring and cementation behaviour of Electrokinetic Stabilisation Technique on Batu Pahat Marine Clay. International Journal of GEOMATE, 11, 2581-2588.

Azhar, A. T. S., Nabila, A. T. A., Shuhaila, M. S., Syalinda, M. Z. N., \& Azmi, M. A. M. (2016b). Electromigration of contaminated soil by Electro-Bioremediation Technique. Soft Soil Engineering Conference 2015 (SEIC 2015). IOP Conference Series: Material Sciences and Engineering 136. IOP Publishing Ltd.
Balia, R. (2018). Old municipal and industrial waste landfills: Examples of possible application of Geophysical Survey Technique for assessment prior to reclamation. Multidisciplinary Journal for Waste Resources \& Residues, 1, 110-115.

Cameselle, C., \& Pena, A. (2016). Enhanced electromigration and electro-osmosis for the remediation of an agriculture soil contaminated with multiple heavy metals. Process Safety and Environment Protection, 104, 209-217. Elsevier.

Chaudhary, N., Singh, B., \& Srivastava, R. K. (2015). Electrokinetic Remediation of heavy metal contaminated soil: A short review. International Daily Journal, 42(191), 6-11. Discovery Publication.

Dixit, R., Wasiullah, Malaviya, D., Pandiyan, K., Singh, U. B., Sahu, A., Shukla, R., Singh, B. P., Rai, J. P., Sharma, P. K., Lade, H., \& Paul, D. (2015). Bioremediation of heavy metals from soil and aquatic environment: An overview of principles and criteria of fundamental processes. Sustainability, 2015, 2189-2212.

Dumont G., Pilawski T., Lenieregue P. D., Hiligsmann S., Delvigne F., Thonart P., Robert T., Nguyen F., \& Hermans T. (2016). Gravimetric water distribution assessment from geoelectrical methods (ERT and EMI) in municipal solid waste landfill. Waste Management, 55, 129-140. Elsevier.

Elicker C., Sanches P. J., \& Castagno K. R. L. (2014). Electroremediation of heavy metals in sewage sludge. Brazillian Journal of Chemical Engineering, 31, 365-371.

Hamdan, A. Z., \& Reddy, K. R. (2018). Transient behaviour of heavy metal in soils during electrokinetic remediation. Science Direct. Chemosphere, 71, 860-871.

Jo, S-U., Kim, D-H., Yang, J-S., \& Baek, K. (2012). Pulsed enhanced electrokinetic restroration of sulfate containing saline greenhouse soil. Electrochimica Acta 86, 57-62. Elsevier. 
Lemly, A. D. (2017). Environmental Hazard Assessment of coal ash disposal at the proposed Rampal Power plant. Human and Ecological Risk Assessment: An International Journal. Taylor\&Francis.

Mosavat, N. (2012). A review of Electrokinetic Treatment Technique for improving the engineering characteristic of Low Permeable Problematic Soils. International Journal of GEOMATE, June 2012, 2(2), 266-272.

Rojo, A., Hansen, H. K., \& Cubillos, M. (2012). Electrokinetic remediation using pulsed sinusoidal electric field. Electrochimica Acta 86, 124-129. Elsevier.

Song, B., Zeng, G., Gong, J., Liang J., Xu, P., Liu Z., Zhang, Y., Zhang, C., Cheng, M., Liu, Y., Ye, S., Yi, H., \& Ren, X. (2017). Evaluation method for assessing the effectiveness of in situ remediation of soil and sediment contaminated with organic pollutants and heavy metals. Environmental International, 105, 43-55. Elsevier.
Sruthy \& Jayalekshmi. (2014). Electrokinetic remediation of heavy metal contaminated soil. International Journal of Structural and Civil Engineering Research.

Suied, A. A., Tajudin, S. A. A., Zakaria, M. N., \& Madun A. (2018). Potential electrokinetic remediation technologies of laboratory scale into field application: Methodology overview. IOP Conferences Series 995: Journal of Physics. IOP Publishing.

Suzuki, T., Moribe, M. Okabe, Y., \& Niinae, M. (2013). A mechanistic study of arsenate removal from artificially contaminated clay soils by electrokinetic remediation. Journal of Hazardous Materials, 254-255. Elsevier. 310-317.

Virtutyte, J., Sillanpaa, M., \& Latostenmaa, P. (2002) Electrokinetic soil remediationcritical overview. The Science of the Total Environment, 289, 97-121. Elsevier. 